\title{
PAR, a protein involved in the cell cycle, is functionally related to chromosomal passenger proteins
}

\author{
MICSUNICA PLATICA ${ }^{1}$, ALIN IONESCU ${ }^{1}$, ELENA IVAN ${ }^{1}$, JAMES F. HOLLAND ${ }^{1}$, \\ JOHN MANDELI $^{2}$ and OVIDIU PLATICA ${ }^{3}$
}

\author{
${ }^{1}$ Department of Medicine, Division of Hematology and Medical Oncology, The Tisch Cancer Institute, \\ ${ }^{2}$ Department of Biomathematical Sciences, Mount Sinai School of Medicine, New York; ${ }^{3}$ Department of Medicine, \\ State University of New York, and VA Harbor Healthcare System, Brooklyn, NY 11209, USA
}

Received October 15, 2010; Accepted November 29, 2010

DOI: 10.3892/ijo.2011.900

\begin{abstract}
Prostate androgen regulated (PAR) protein is a 148 amino acid polypeptide ubiquitously expressed in normal cells and overexpressed in many malignancies. Manipulation of PAR mRNA in DU145 and NIH3T3 cells indicated that its expression level is an important determinant of cell in vitro proliferation, clonogenicity in soft agar and in vivo tumorigenicity. In this study, we showed that PAR is a short-lived protein with a peak in $\mathrm{G} 2 / \mathrm{M}$ phase. Using immunofluorescent antibodies we showed that PAR moves from centrosomes in prophase and metaphase to spindle midzone in anaphase, and concentrates to midbody in telophase and cytokinesis. During mitosis a fraction of PAR can also be detected in the cytoplasm. PAR pattern of expression and its dynamic localization suggested a functional relationship to chromosomal passenger proteins (CPP). This protein colocalized with Aurora A at centrosomes in metaphase, and with survivin at midbody in telophase and cytokinesis. It also formed complexes with Aurora A, and with survivin, Aurora B and INCENP. In addition, PAR increased Aurora B kinase activity on histone H3. The decreased PAR levels in DU145 cells resulted in defects in centrosome segregation, in failed cytokinesis and chromosome alignment, and in increased number of apoptotic cells, polyploidy and aberrant mitosis. It is known that such defects could lead to genomic instability and tumorigenesis. In this study we also confirm our earlier findings that PAR is overexpressed in many tumors. Due to its involvement in cell cycle and its overexpression in several human cancers PAR could represent an attractive target for therapeutic intervention.
\end{abstract}

Correspondence to: Dr Ovidiu Platica, Department of Medicine, State University of New York, and VA Harbor Healthcare System, Brooklyn, NY 11209, USA

E-mail: ovidiu.platica@va.gov

Key words: prostate androgen regulated, JTB, chromosomal passenger proteins, cell cycle

\section{Introduction}

Cellular division is a process which requires a temporal and spatial well-coordinated machinery. Defects in this mechanism can lead to genomic instability, which in turn could result in cell death or cancer (1-3). During the past few years considerable progress has been made towards the understanding the structure and function of the factors involved in cell division. Many mitotic regulators, like chromosomal passenger proteins (4-6) have been identified, but their involvement in mitosis is not yet fully understood. Knowledge of the structure and function of these proteins will allow the control of cell cycle regulatory machinery.

We previously reported the identification by substraction of a gene designated PAR (prostate androgen regulated) (7) from $\mathrm{LNCaP}-\mathrm{OM}$ cells, an androgen resistant prostatic carcinoma subline (8). The JTB, jumping translocation gene, with a similar nucleotide sequence was reported by Hatakeyama et al (9). The $3 \mathrm{~kb}$ PAR gene encodes 5 exons and is located on chromosome 1q23 in the epidermal differentiation complex. The PAR cDNA has 1038 bp and encodes a polypeptide of 146 amino acids with an estimated MW of $16 \mathrm{kDa}$ (accession no. AF115850).

PAR is ubiquitously expressed in normal tissues and is overexpressed in many malignancies. Manipulation of PAR mRNA in DU145 human androgen resistant prostate cancer cells and NIH3T3 mouse embryonic fibroblast cells indicated that the level of its expression is an important determinant of proliferation rate in vitro, clonogenicity in soft agar, and tumorigenicity in vivo $(10,11)$. This relationship between cell proliferation and PAR expression suggested a potential role for PAR in the cell cycle.

In this study we investigated the role of PAR in the cell cycle and its involvement in malignant transformation. The data presented herein demonstrate that PAR is a cell cycle regulated protein with a pattern of expression and localization suggestive a functional relationship to chromosomal passenger proteins (CPP). Furthermore, we provide evidence that PAR may be an activator of Aurora kinase. Finally, we confirm and extend our earlier finding that PAR is overexpressed in several human tumors. 


\section{Materials and methods}

Cell lines. The following cell lines were used in this study: human breast adenocarcinoma MCF7 and T47D, human prostate carcinoma DU145 and $\mathrm{LNCaP}$, human normal adult fibroblast NHF, human normal breast MCF10F, normal prostate epithelial RWPE-1. All cell lines were obtained from the American Type Culture Collection (Manassas, VA), except for NHF, which was obtained from Fisher Scientific (Pittsburg, PA). The cells were maintained at $37^{\circ} \mathrm{C}$ in a $5 \%$ $\mathrm{CO}_{2}$ atmosphere as recommended by the manufacturer.

Cell cycle synchronization. Cells were synchronized at the G1/S phase using double thymidine incorporation (SigmaAldrich). Cells were seeded at a density of $1 \times 10^{6}$ in a $60 \mathrm{~mm}$ dish and cultured in $10 \%$ FBS supplemented medium for $24 \mathrm{~h}$. The cells were then cultured for an additional $19 \mathrm{~h}$ in the presence of $2 \mathrm{mM}$ thymidine (Sigma-Aldrich, St. Louis, MO), washed three times with a $1 \mathrm{X}$ phosphate-buffered saline (PBS) solution, and transferred to normal growth medium. After 9 h $2 \mathrm{mM}$ thymidine was added and incubation was continued for an additional $16 \mathrm{~h}$. The cells were washed with PBS and placed in normal fresh medium.

Flow cytometry. The percentage of cells in different phases of the cell cycle at various time-points was quantified by flow cytometry analysis (Becton-Dickinson FACS StarPlus) as previously described $(10,11)$. Briefly, $1 \times 10^{6}$ cells were trypsinized, washed with PBS, fixed in $70 \%$ ethanol, treated with $500 \mu \mathrm{g}$ DNase free RNase A for $30 \mathrm{~min}$ at $37^{\circ} \mathrm{C}$, stained with propidium iodide $(250 \mu \mathrm{g} / \mathrm{ml})$ and analyzed in a Beckman-Dickinson flow cytometer. The cell distribution pattern was assessed using CellQuest software. The synchronized cells were harvested at various intervals of time after the release from the second thymidine block. Extracts were prepared and analyzed by Western blot analysis.

Northern blot analysis. A Cancer Profiling Array (Clontech) which includes normalized cDNA ( $2 \mu \mathrm{g}$ polyA RNA) from 247 tumors (breast, uterus, colon, stomach, ovary, lung, prostate, kidney, rectum, thyroid, small intestine) and corresponding normal tissues from the same patient was analyzed for PAR expression. After prehybridization, the blot was hybridized to the PAR encoding sequence labeled with ${ }^{32} \mathrm{P}$ by the random primer method, as previously described (7). The autoradiograms were read with a flatbed scanner UMAX Astra 1200 and the intensity of the bands compared with NIH Image software version 1.61, as described $(10,11)$.

Western blot analysis. Preparation of cellular lysates, SDSpolyacrylamide electrophoresis, and transfer to nitrocellulose paper was performed as previously described (12). Briefly, the cells were sonicated at $4^{\circ} \mathrm{C}$ in lysis buffer $(1 \%$ Nonidet P-40, 50 mM Tris (pH 7.5), 1 mM EGTA, 1 mM dithiothreitol (DTT), $120 \mathrm{mM} \mathrm{NaCl}, 5 \mathrm{mM}$ EDTA, protease inhibitor cocktail (Calbiochem, La Jolla, CA), $5 \mathrm{mM} \mathrm{NaF}, 0.1 \mathrm{mM}$ sodium vanadate). Protein concentration was determined with the Bio-Rad protein assay. Lysates containing 20-30 $\mu \mathrm{g}$ protein were separated in a 10-12\% SDS-polyacrylamide gel, transferred to nitrocellulose membranes (Schleicher and
Schuell, Keene, NH) and immunoblotted with appropriate antibodies. The blots were processed using Supersignal West Pico Substrate and exposed to CL-XPosure film from Pierce Company (Rockford, IL). The film was read with a flatbed scanner (Epson Perfection 1650) and the intensity of the bands was compared with NIH Image software. For normalization the blots were stripped and reprobed with anti- $\beta$ actin antibody (Sigma, Milwaukee, WI).

Antibodies. The following antibodies were used: a highly specific affinity purified rabbit polyclonal antibody raised against PAR peptide P1 (RQRQLDRKALEKVRK) selected from PAR carboxy-terminal domain (ResGen/Invitrogen, Huntsville, AL); polyclonal and monoclonal anti-Aurora-A (Cell Signaling Technology, Beverly, MA); polyclonal antiAurora B (Invitrogen, Carlsbad, CA); polyclonal anti-INCENP (Life Technologies Corp., Carlsbad, CA); polyclonal anti-H3 and pSer-10 histone $\mathrm{H} 3$ antibodies (Upstate Biotechnology, Lake Placid, NY); anti-survivin and anti-E-cadherin monoclonal antibody (Santa Cruz Biotechnology, CA), and anti-ß3actin polyclonal antibody (Invitrogen).

Immunoprecipitation. DU145 cells were lysed in $1 \mathrm{ml}$ of lysis buffer. Proteins were immunoprecipitated using the Seize $\mathrm{X}$ Protein A kit (Pierce) according to the manufacturer's instructions. The antibody of interest (50-500 $\mu \mathrm{g})$ was immobilized to the Protein A, then, the DSS cross linker was added. In the next step the gel containing the bound antibody was washed and incubated overnight with the sample containing 250-500 $\mu \mathrm{g}$ of protein mixture. After extensive washing with Wash Buffer, the immunoprecipitated antigen was eluted and analyzed by SDS-PAGE as previously described (12).

Immunofluorescence. Cells $\left(1 \times 10^{5}\right)$ were grown in BD Falcon 4 chamber polystyrene culture slides (Franklin Lakes, NJ), fixed in $100 \%$ methanol previously cooled at $-20^{\circ} \mathrm{C}$ for $10 \mathrm{~min}$ and permeabilized for 2 min with $0.1 \%$ Triton X-100 in PBS. After blocking for $1 \mathrm{~h}$ in $5 \%$ goat serum and 5\% glycerol in 1X PBS- $0.1 \%$ Triton X-100, the slides were incubated with the indicated antibody for $1 \mathrm{~h}$ at room temperature (rabbit anti-PAR antibody, 1:200; anti-Aurora-A antibody, 1:250; anti-Aurora-B antibody, 1:100; anti-survivin antibody, 1:200). The slides were then washed with 1 XPBS and incubated for $1 \mathrm{~h}$ at room temperature with a secondary antibody (Alexa Fluor dye labeled anti-mouse, anti-rabbit or anti-goat (Invitrogen/ Molecular Probes). Immunostained samples were then washed and counterstained for 2 min with 4',6-diamidino-2phenylindole dihydrochloride (DAPI; Boehringer-Ingelheim, Germany), before being subjected to confocal microscopy. Image collection was done on a DMLB Leica (Wetzlar, Germany) microscope.

In vitro aurora kinase assay. Assays were performed following the Kinase Assay Protocol (Upstate Cell Signaling Solutions, Temecula, CA). All the required stock solutions, Tris assay dilution buffer, Histone H3, Aurora B active and Magnesium/ ATP cocktail, were purchased from the same company. Briefly, mixtures containing Tris Dilution Buffer, Histone H3, Aurora B active, PAR recombinant protein and Magnesium/ 
ATP cocktail were incubated for $10 \mathrm{~min}$ at $37^{\circ} \mathrm{C}$. The samples were boiled for $5 \mathrm{~min}$ and analyzed by Western blot analysis as described (12).

Transfection with antisense oligonucleotides. A 22 mer antisense phosphothionated oligonucleotide (5'-agg tac gaa cgc cca cgg ccc t-3') complementary to the first 19 nucleotides of the PAR encoding sequence, and an oligonucleotide with the same sequence but containing 4 scrambled bases, were synthesized by the Midland Certified Reagent Company (Midland, TX). A BLASTN search in GenBank showed no homology of these oligonucleotides with any other known gene sequences.

The DU145 cells were transfected with $2 \mu \mathrm{M}$ oligonucleotides using lipofectin as described (10). Briefly, 1x105 DU145 cells were cultured in DMEM medium supplemented with $10 \% \mathrm{FCS}$ in $60 \mathrm{~mm}$ tissue culture dishes at $37^{\circ} \mathrm{C}$ in $5 \% \mathrm{CO}_{2}$ atmosphere until they reached $\sim 50 \%$ confluence. The cells were then washed with serum-free DMEM and overlaid with $2 \mathrm{ml}$ serum free OPTI-MEM medium containing $10 \mu \mathrm{l}$ of Lipofectin (Life Technologies Inc., Gaithesburg, MD) and $2 \mu \mathrm{M}$ oligonucleotides. After $5 \mathrm{~h}$ of incubation at $37^{\circ} \mathrm{C}$, the medium was replaced with $4 \mathrm{ml}$ DMEM supplemented with $10 \%$ FCS and incubation was continued for an additional $48 \mathrm{~h}$. The transiently transfected cells were analyzed for PAR expression by Western blotting as described described (12).

Statistical methods. PAR mRNA expression was measured in pairs of human malignant and normal adjacent tissue, collected from different organs. For each pair, the probability of PAR mRNA overexpression in malignant tissue compared with normal tissue was determined by McNemar's test using 0.05 level of significance.

\section{Results}

PAR expression undergoes changes during cell cycle progression. To study PAR protein expression during the cell cycle we used the DU145 human prostate cancer cells and MCF7 human breast cancer cells, synchronized by double thymidine incorporation in G1/S phase (Fig. 1A). The cell cycle stage was monitored by flow cytometry analysis (Fig. 1B). Densitometric quantification of PAR protein level and percentage of cells in $\mathrm{G} 2 / \mathrm{M}$ phase is presented in Fig. 1C.

Western blot analysis with anti-PAR P1 antibody shows that PAR protein level changed during the progression through the cell cycle. Its level was lowest at the G1/S transition, gradually increased as the cells progressed through $\mathrm{S}$ phase, reached the highest level in G2 and in mitosis, and returned to low levels as the cells re-entered G1. The band indicating the PAR protein in Western blot analyses was completely blocked when the antibody was pre-incubated with peptide P1. Similar results were obtained with protein lysates prepared from DU145 and MCF7 cells synchronized by starvation and released (data not shown).

PAR localization changes during cell mitosis. To investigate PAR protein localization during the mitosis we utilized immunofluorescent anti-peptide P1 antibody. Fig. 2 shows the distribution of PAR during the mitosis in asynchronously
A

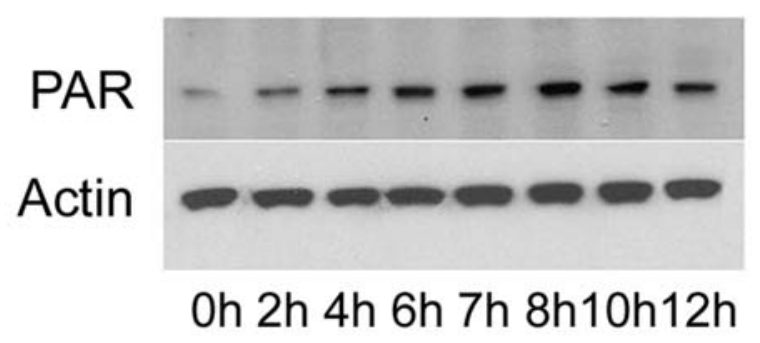

B

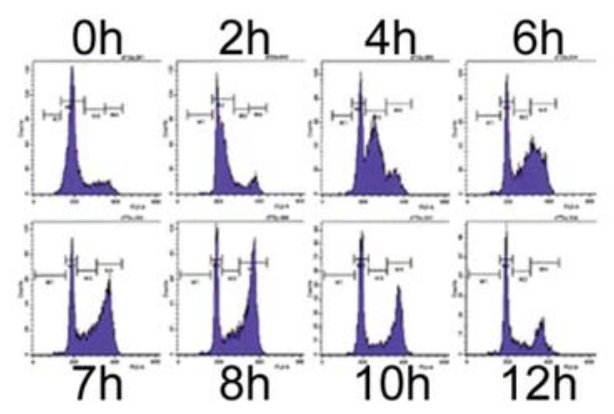

C

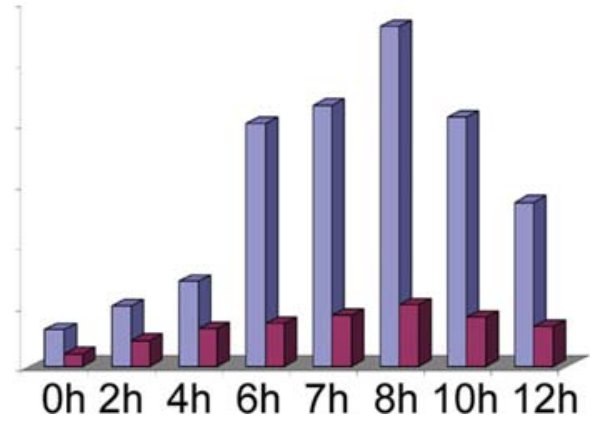

Figure 1. Cell cycle-regulated PAR expression. (A) PAR expression in DU145 cells synchronized by double thymidine block at $0,2,4,6,7,8,10$, and $12 \mathrm{~h}$ after thymidine release. (B) Flow cytometry analysis of DNA content of DU145 cells synchronized by double thymidine block. (C) Densitometric reading of PAR protein level during the cell cycle (black) and percentage of cells in G2/M phase of the cell cycle (blue).

growing DU145 cells. During prophase (Fig. 2A) and metaphase (Fig. 2B) PAR is localized in the centrosomic region and along the spindle fibers. PAR moves to the spindle midzone at the onset of anaphase (Fig. 2C) and concentrates at the midbody in late telophase and cytokinesis (Fig. 2D and E). In addition, during prophase and later stages of mitosis a cytoplasmic fraction of PAR protein could be detected. This pattern of PAR localization was completely blocked when the antibody was preincubated with peptide P1 (Fig. 3). A similar pattern of localization was also observed in NHF human fibroblasts and MCF7 breast tumor cells (data not shown).

PAR is functionally related with chromosomal passenger proteins. The dynamic changes in localization during cell cycle suggested that PAR might be functionally related to the chromosomal passenger protein family, which includes INCENP (13), survivin (14-16), Aurora (17-19), Borealin (20), and TD-60 (21) proteins. To explore this hypothesis we first investigated whether PAR colocalizes with chromosomal 


\section{A}
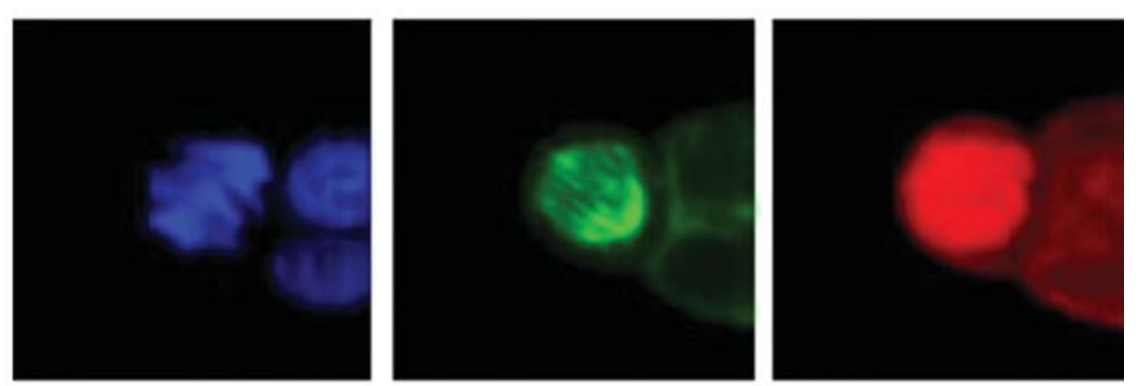

B
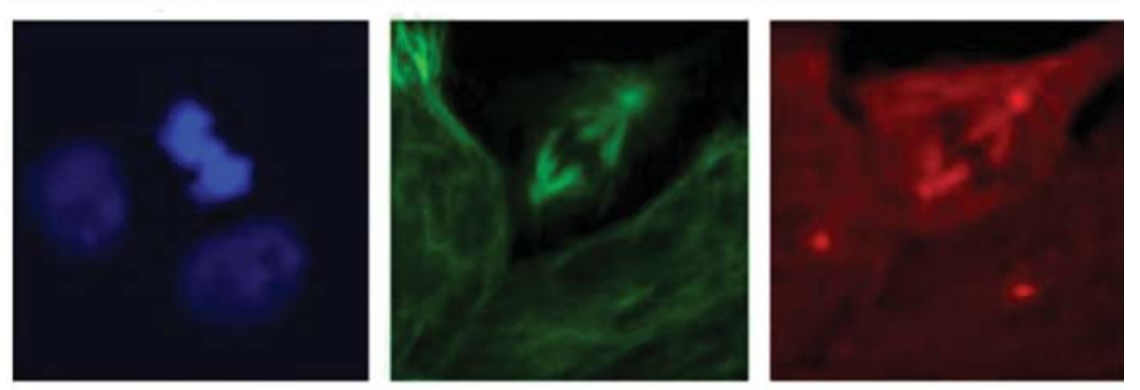

C
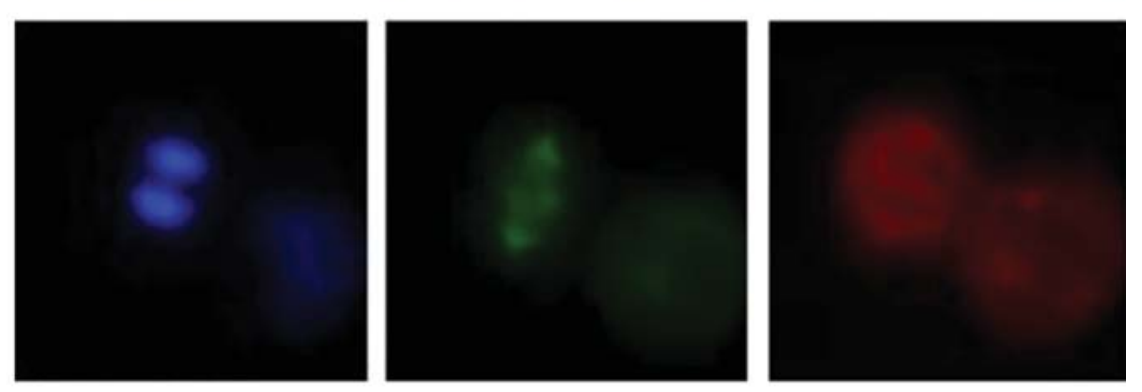

D
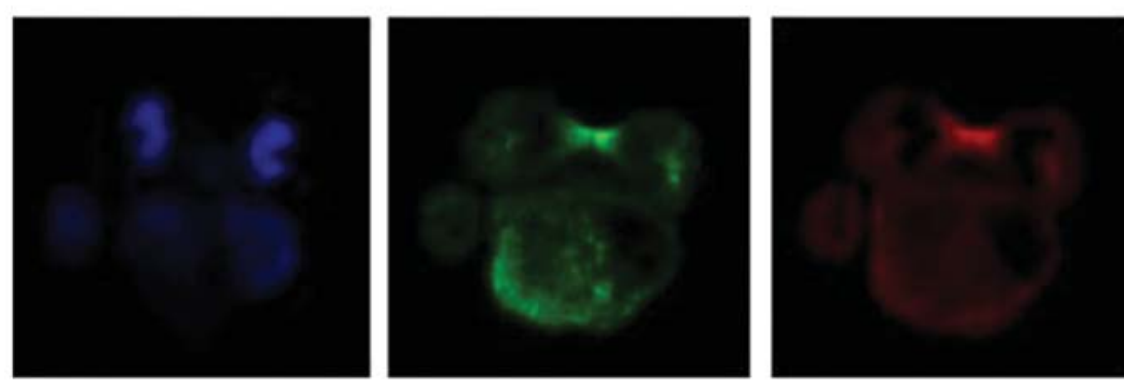

E
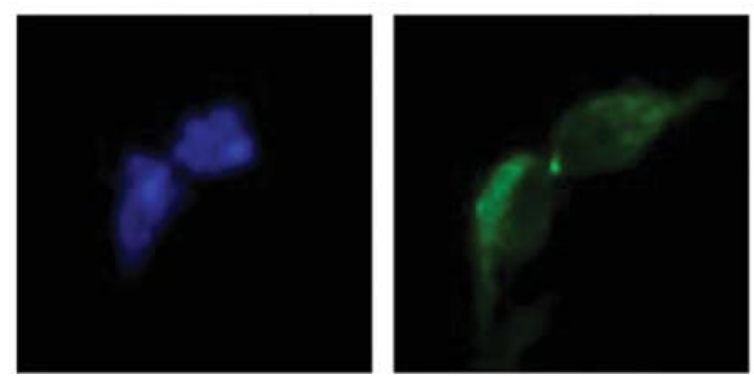

Figure 2. Simultaneous immunofluorescence staining for PAR and B-tubulin of DU145 cells during mitosis. (A) Prophase, (B) metaphase, (C) anaphase, (D) telophase, and (E) midbody telophase/ cytokinesis. Tubulin (green); PAR (red). DNA stained with 4, 6-diamidino-2- phenylindole DAPI (blue).

passenger proteins. Immunostaining with antibodies to Aurora A, survivin and peptide P1 showed that PAR colocalizes with Aurora A at centrosomes during prophase and metaphase (Fig. 4A), and with survivin at the midbody in telophase (Fig. 4B).

Next we looked for PAR possible physical interaction with chromosomal passenger proteins. Initially, we looked for possible complexes between PAR and Aurora A. Total lysates from DU145 cells immunoprecipitated with anti-Aurora A antibody were analyzed by Western blotting with anti-Aurora A and anti-PAR antibodies. Bands characteristic for Aurora A and PAR were detected (Fig. 5). When the same blot was immunoblotted with anti E-cadherin antibody no band could be detected, indicating that PAR interacts specifically with Aurora A. Similarly, lysates of synchronized DU145 cells, immunoprecipitated with anti-PAR antibody and analyzed by Western blotting showed bands characteristic of PAR and Aurora A. Preincubation of the immunoprecipitating anti- 
1

A

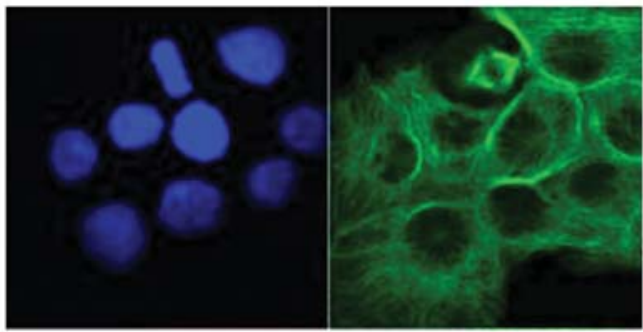

B

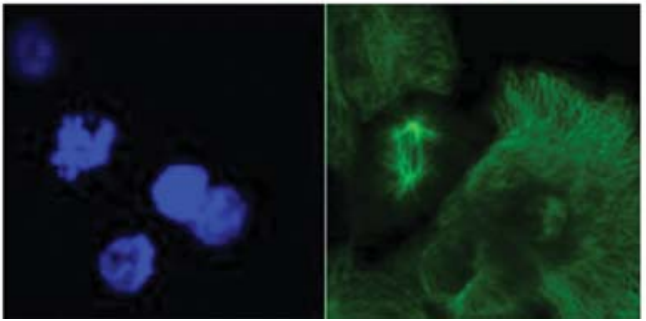

3
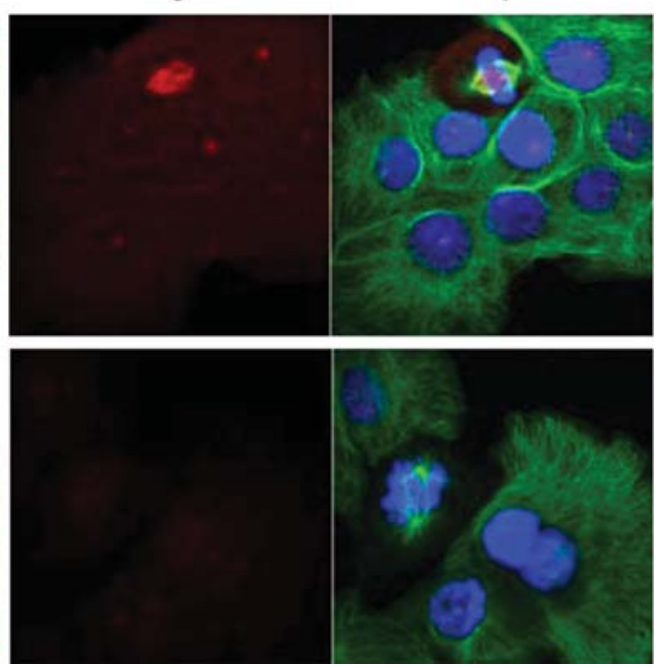

Figure 3. PAR localization blocked when PAR antibody was preincubated with peptide P1. (A) PAR stained with anti-PAR antibody; (B) PAR stained with blocked anti-PAR Ab. 1, DNA (blue); 2, tubulin (green); 3, PAR (red); 4, composite pictures.
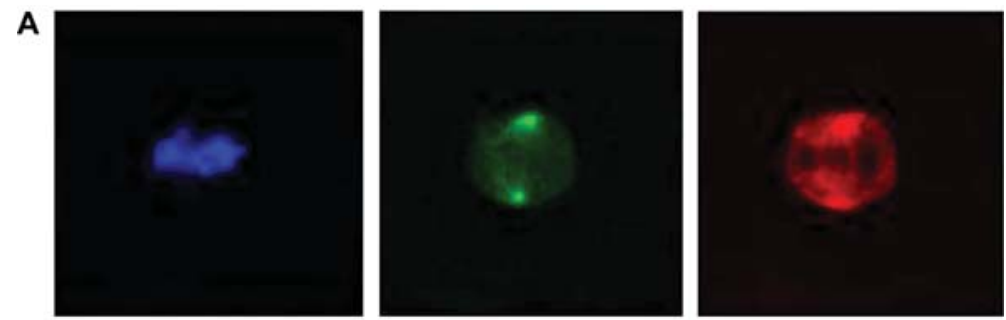

B
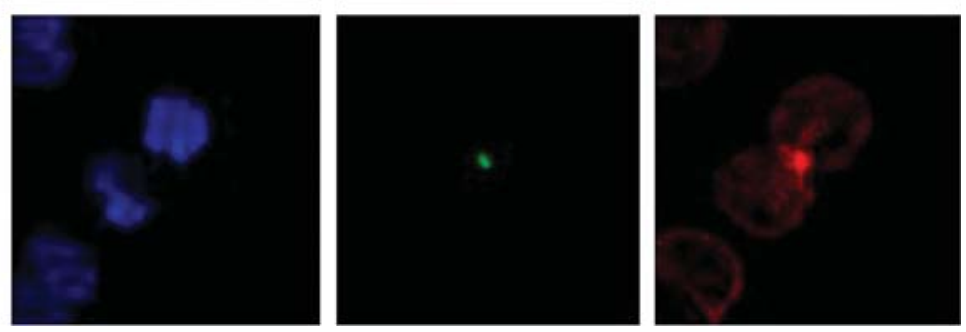

Figure 4. (A) PAR colocalization with Aurora A at centrosomes in DU145 cells. Aurora A (green) and PAR (red), DNA (blue). (B) PAR colocalization with Survivin at midbody. Survivin (green), PAR (red), and DNA (blue).

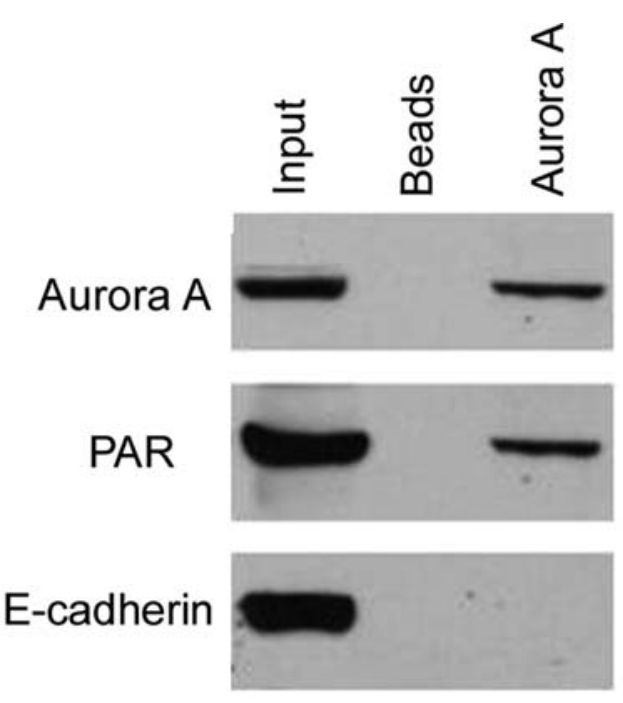

Figure 5. PAR and Aurora A are physically associated. The immunoprecipitated protein complexes were analyzed by Western blotting using anti-PAR and Aurora A antibodies.
PAR antibody with the peptide P1 abolished both bands. No bands were observed when immunoprecipitation was performed with beads alone.

To investigate whether PAR interacts with survivin, lysates from DU145 immunoprecipitated with anti-survivin antibody were analyzed by Western blot analyses. Bands characteristic for survivin and PAR were observed when the blots were treated with the appropriate antibodies (Fig. 6). In addition, these immunoprecipitates showed bands characteristic for Aurora B and INCENP when immunoblotted with the proper antibodies, suggesting that PAR is probably a component of the complex already described involving Aurora B, survivin, Borealin, and INCENP (22-25).

PAR changes Aurora B kinase activity. Since it has been reported that Aurora is the only chromosomal passenger protein which has mitotic serine threonine kinase activity $(17,18,26)$, we explored the effect of PAR on Aurora kinase activity. As shown in Fig. 7, in the presence of PAR protein Aurora B-phosphorylation of histone H3 increased, suggesting 


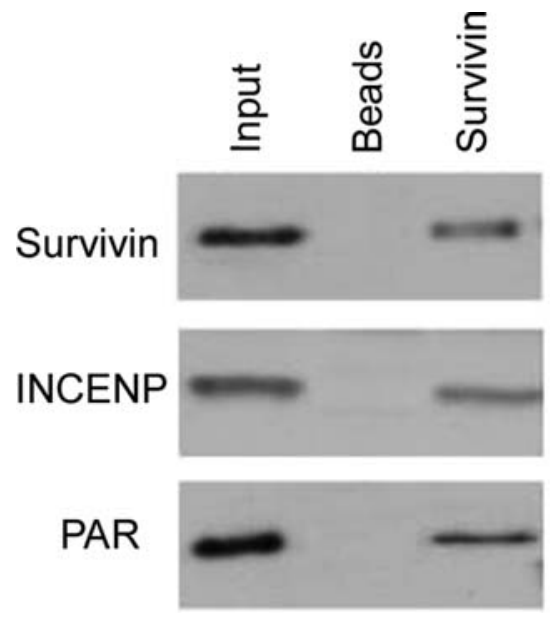

Figure 6. PAR, Aurora B, Survivin and INCENP form a complex.

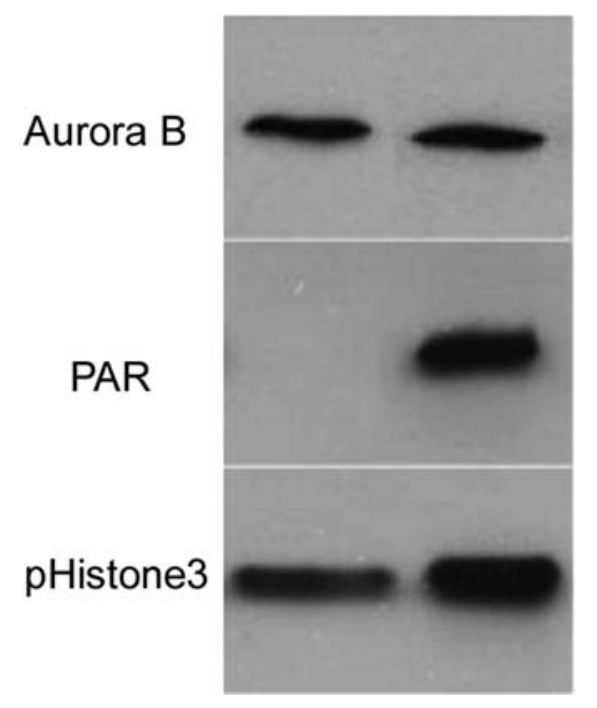

Figure 7. PAR enhances Aurora-B kinase activity in vitro. Aurora-B was incubated with histone $\mathrm{H} 3$ in the presence or absence of PAR. The reactions were resolved on the SDS-PAGE gel and immunoblotted with anti-histone H3 antibody.

that PAR could be an activator of this kinase. Conversely, when cyclin B was added no stimulation of kinase activity was observed.

PAR inhibition leads to defects during mitosis. To assess the role of PAR during mitosis we examined the effect of modulation of PAR expression on DU145 human prostate cancer cells. Western blot analysis of transiently transfected asynchronously growing DU145 cells with PAR antisense oligonucleotides resulted in decreased PAR levels compared to untransfected cells, by $48 \mathrm{~h}$. In contrast, $B$-actin protein level did not show any changes in transfected cells. The transfection with scrambled oligonucleotide did not change the PAR or B-actin level (Fig. 8). These results indicate that inhibition of PAR expression was due specifically to the antisense oligonucleotide transfection.

At $48 \mathrm{~h}$ after antisense oligonucleotide transfection DU145 cells displayed complex defects at various stages of cell cycle like multipolar spindles, multiple nuclei, lagging chromosomes

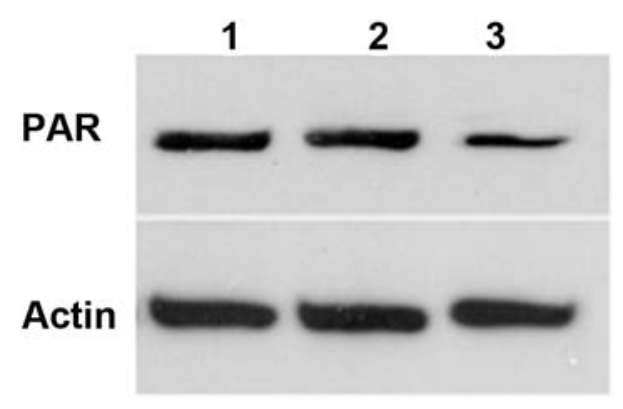

Figure 8. Expression of PAR protein following transient transfection of DU145 cells. 1, control untreated DU145 cells; 2, DU145 cells treated with scrambled oligonucleotides; 3 , DU145 cells transfected with $2 \mu \mathrm{M}$ oligonucleotides in the presence of lipofectin. Cells were harvested, and PAR protein was analysed by Western blot analysis.

Table I. PAR mRNA is overexpressed in various malignancies.

\begin{tabular}{lccc}
\hline Tissue & Overexp. no./total no. & $\%$ & p-value \\
\hline Breast & $43 / 52$ & 82.6 & $<0.001$ \\
Ovary & $15 / 16$ & 93.7 & $<0.001$ \\
Lung & $17 / 21$ & 80.9 & $<0.01$ \\
Uterus & $33 / 44$ & 75 & $<0.001$ \\
Colon & $26 / 37$ & 70 & $<0.05$ \\
Rect & $12 / 18$ & 66.6 & $\mathrm{NS}$ \\
Thyroid & $4 / 6$ & 66.6 & \\
Prostate & $3 / 4$ & 75 & \\
Stomach & $14 / 27$ & 51 & \\
Kidney & $6 / 20$ & 30 & \\
Small intestine & $0 / 2$ & & \\
\hline
\end{tabular}

Overex no./total no., number of specimens with PAR overexpressed over the total number of specimens from each category examined. $\%$, percentage of overexpressed specimens from the total of specimens from each category examined. NS, not significant.

in anaphase, increased number of apoptotic cells, increased polyploidy and aberrant mitosis (Fig. 9).

PAR is overexpressed in malignancies. We previously reported that PAR RNA is overexpressed in various malignant cell lines and tissues compared to their normal counterparts (7). To further explore PAR mRNA expression, a dot blot containing pairs of human malignant and normal adjacent tissues, collected from different organs was examined. As shown in Table I, PAR mRNA was significantly overexpressed in various malignant tissues, including breast $(43 / 52, \mathrm{p}<0.001)$, ovary $(15 / 16, p<0.001)$, lung $(17 / 21, \mathrm{p}<0.01)$, uterus $(33 / 44$, $\mathrm{p}<0.001)$, colon $(26 / 37, \mathrm{p}<0.05)$, and rectum $(12 / 18$, nonsignificant) compared to the normal tissues.

PAR protein was also overexpressed in the MCF7 and T47D human breast cancer cells and the DU145 and LNCaP human prostate cancer cells compared to normal cell lines MCF10 and RWPE-1, as determined by Western blot analysis (Fig. 10). 

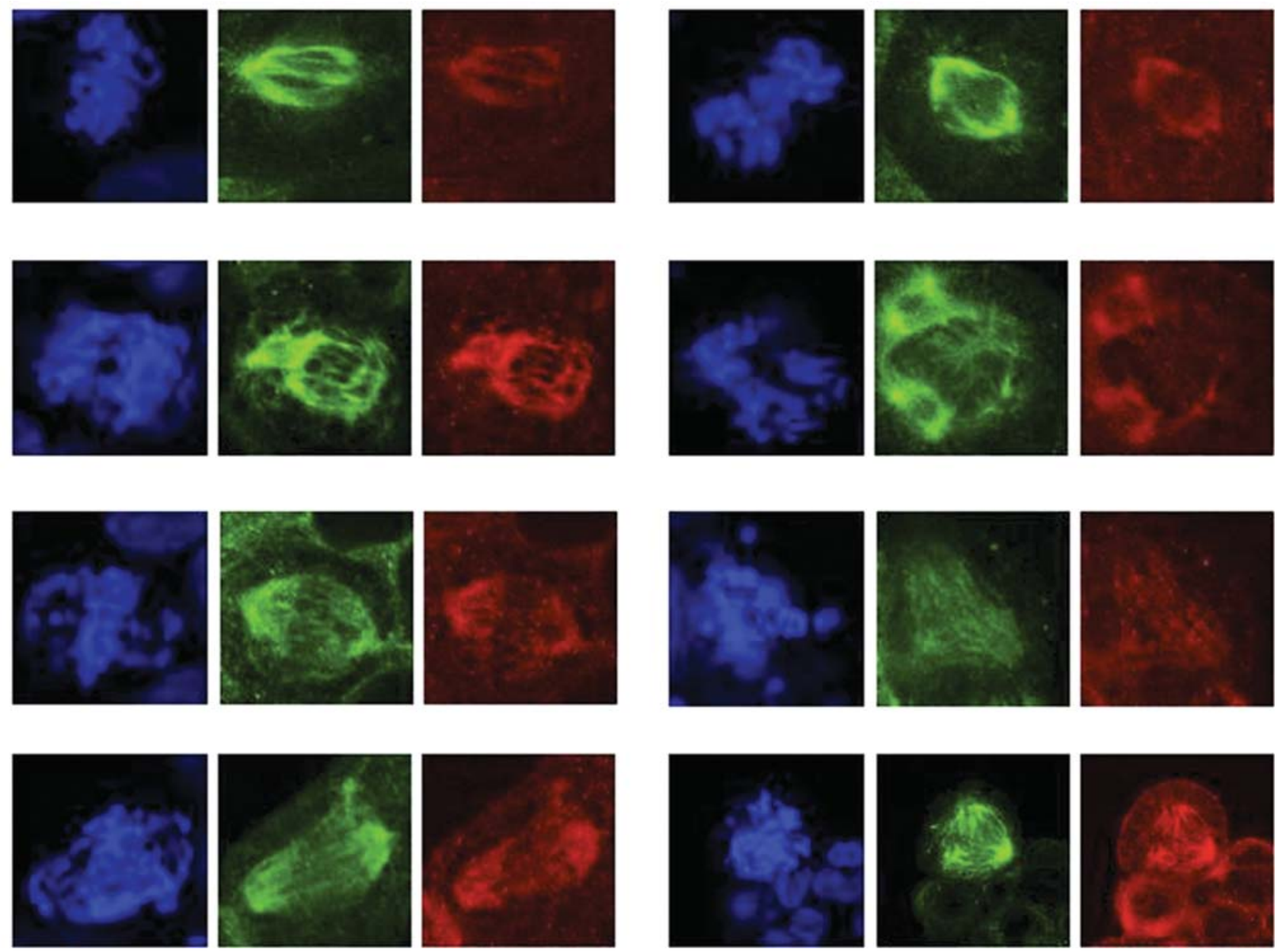

Figure 9. Asynchronous DU145 cells transiently transfected with anti oligo PAR showing centrosome separation aberrations, spindle aberrations, cells with multipolar spindles, aberrant mitosis, failure to align chromosomes, by $48 \mathrm{~h}$ (blue, DAPI; green, anti-tubulin; red, anti-PAR).

MCF10F MCF7 T47D RWPE-1 LNCaP DU145

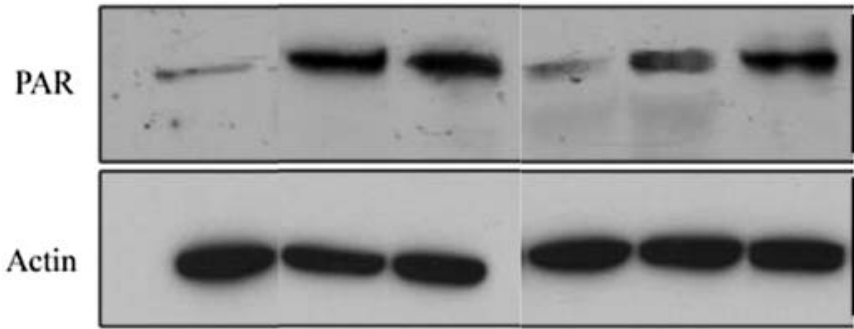

Figure 10. PAR protein is overexpressed in human breast and prostate cancer cell lines. Lysates (30 $\mu \mathrm{g}$ protein) from human breast cancer cell lines (MCF7 and T47D) and human prostate cancer cell lines (LNCaP and DU145), normal breast cells MCF10F, and normal prostate cells RWPE-1 were analysed on SDS-PAGE and immunoblotted with anti-PAR antibody. The Western blotting was also probed with anti-ß-actin for a loading control.

\section{Discussion}

The PAR gene is ubiquitously expressed in normal tissues. Its level of expression is higher in rapidly dividing cells and is significantly upregulated in a broad range of human cancers, suggesting its involvement in proliferation (7). In addition, PAR possesses oncogenic activity. Ectopic expression of PAR in NIH3T3 cells induced malignant transformation (11).
The data presented here indicate that PAR is a cell cycle regulated protein. Its expression is lowest at $\mathrm{G} 1 / \mathrm{S}$, peaks in $\mathrm{G} 2$ and mitosis, and returns rapidly to a low level at the beginning of G1, indicating that PAR is probably degraded upon exit from mitosis. The presence of $2 \mathrm{D}$ boxes RxxL and RxxLxxV at the amino- and carboxy-terminus respectively in the PAR amino acid sequence, suggests that PAR degradation can occur through the ubiquitin-proteasome pathway mediated by the anaphase-promoting complex (APC). This observation is in line with reports that a number of other proteins involved in the regulation of mitosis, including Aurora kinases, are degraded when entering the G1 phase by the ubiquitin proteasome pathway (27-29). The timely destruction of PAR is probably important for normal cells since high level of PAR is associated with increased cell proliferation and malignant phenotype $(7,11)$.

PAR localization shows a dynamic distribution during the cell cycle moving from the centrosomes in interphase, prophase and metaphase, to the spindle midzone at the onset of anaphase, and finally to the midbody in telophase and cytokinesis. This distribution parallels that of chromosomal passenger proteins, which have an important role in centrosome and chromosome segregation, spindle assembly and completion of cytokinesis (19,30-33). CPP associate with centrosomes and chromosomes early in mitosis, are transfered to the 
spindle midzone at anaphase, and localize at the midbody in telophase and cytokinesis.

Additional evidence of a functional relationtionship between PAR and CPP comes from the data showing that immunostained endogenous PAR is sequentially colocalized with Aurora A at centrosomes in metaphase, and with survivin at the midbody in telophase and cytokinesis.

Aurora is the only chromosomal passenger protein which is a mitotic serine-threonine kinase $(18,34,35)$. The kinase has multiple activators like Ajuba (36,37), which is localized to the centrosome and probably, activates the kinase during mitotic entry, or TPX2 (38-40), which is located on the mitotic spindle and activates the kinase on spindle microtubules. However, it has been suggested that other factors, should be accounted for as activators of Aurora kinase activity from late G2 to metaphase (41). Our data suggest that PAR could be one of these activators. In the presence of PAR, Aurora phosphorylated histone $\mathrm{H} 3$, a physiological substrate of Aurora kinase which is phosphorylated at serine 10 only in mitosis (42-44), more efficiently than in its absence.

The subcellular localization of PAR suggests its possible function. During prophase and metaphase PAR is located in the centrosomes, the major microtubule-organizing centers of the cell, which are involved in bipolar spindle formation, chromosome segregation, and cytokinesis. They are critical for genomic stability in the cells (45-47). Later on, in telophase and cytokinesis PAR is found at the midbody, a dense transient structure containing microtubules derived from the spindle midzone tightly bundled by the cytokinetic furrow. The midbody contains proteins essential for chromosome segregation, symmetric cell division, cytokinesis and for coordinating the transition from $\mathrm{M}$ to $\mathrm{G} 1$ phase of the cell cycle $(48,49)$.

Cytokinesis is one of the most important phases of cell division when the chromosomes, cytoplasm, and organelles are equally divided to daughter cells. Defects in this process cause also genome instability and predisposes to cancer $(50,51)$.

Thus, PAR, being sequentially localized at places with a key role in cell cycle, and also being functionally related with chromosomal passenger proteins, could play a significant role in various aspects of the cell division like centrosome replication and segregation, spindle formation and cytokinesis. Lower PAR levels induced by transient transfection with PAR antisense oligonucleotides into DU145 cells strongly disrupted the course of these mitotic events. The resulting phenotypes showed defects at multiple stages of the cell cycle: defects in centrosome segregation (cells with multipolar spindles), in cytokinesis (large number of multinucleated cells), defects in chromosome alignment (lagging chromosomes), increased number of apoptotic cells, increased polyploidy and other morphologic abnormalities. These alterations could lead to genomic instability resulting in tumorigenesis or cell death. Our previously reported work with DU145 cells stably transfected with anti-PAR sequence (10) showed that prolonged periods of reduced PAR expression caused a decrease in cell proliferation, arrest in G2/M and increased cell death. PAR could function independently in centrosome segregation, spindle formation and cytokinesis.

Alternatively, PAR could work by being complexed with chromosomal passenger proteins. Co-immunoprecipitation studies have demonstrated the interaction of PAR with these proteins. PAR could form a complex with Aurora A or with Aurora B, survivin, INCENP, TD60 and Borealin in a complex already described (51-54). It was reported that interference with the function of survivin, INCENP or Aurora B or Borealin leads to defects of mitosis and cytokinesis similar to those observed with induced lower levels of PAR. Lastly, silencing PAR could cause a major defect in centrosome duplication, leading to aberrant mitosis or other downstream effects.

Since PAR was overexpressed in the initial screening of several breast and prostate cancer cell lines and tissues we examined PAR expression in a panel of 52 human breast tumors and their adjacent normal counterpart. More than $80 \%$ of the malignant breast tissues significantly overexpressed PAR mRNA compared to the adjacent normal tissues. Similarly, PAR was significantly overexpressed in ovary $(93.7 \%, n=16)$, lung $(81 \%, n=21)$, uterus $(75 \%, n=44)$, colon $(70 \%, n=37)$, rectum $(67 \%, n=18)$ compared to the normal counterparts. Recently, Wong et al (56) reported a significant overexpression of JTB (PAR) in liver tumors compared to the paired adjacent normal liver tissues. However, Kanome et al (57) using commercial dot blots found JTB expression decreased in lung, kidney, stomach and colon cancers compared to normal counterparts.

PAR overexpression could be due to gene amplification since PAR is localized on chromosome $1 q 21$, a region commonly amplified in many malignancies including sarcomas, breast cancer and other solid tumors. However, other mechanisms such as, enhanced gene transcription, gene rearrangement, or abnormal stability of PAR protein could also explain PAR overexpression.

Although PAR is overexpressed in many cancers the mechanism that links PAR to tumor development is not fully understood. Alterations in PAR expression and localization could lead to abnormal PAR activity and interaction with other cellular proteins. This can contribute to the dysregulation of the cell cycle progression and ultimately results in malignant transformation. The translocation of a part of JTB (PAR) has been associate with leukemia (9). Studies are in progress to better define the PAR involvement in tumor development. If these studies confirm our previous findings, alteration of PAR level could be a marker for malignant cell phenotype. Due to its involvement in the cell cycle and its overexpression in many human cancers PAR could represents an attractive target for therapeutic intervention.

\section{Acknowledgements}

This work was supported by the T.J. Martell Foundation for Leukemia, Cancer, and AIDS Research.

\section{References}

1. Giet R, Petretti C and Prigent C: Aurora kinases, aneuploidy and cancer, a coincidence or a real link. Trends Cell Biol 15: 241-250, 2005.

2. Kops GJ, Weaver BA and Cleveland DW: On the road to cancer: aneuploidy and the mitotic checkpoint. Nat Rev Cancer 5: 773-785, 2005.

3. Malumbres $\mathrm{M}$ and Barbacid M: To cycle or not to cycle: a critical decision in cancer. Nat Rev Cancer 1: 222-231, 2001. 
4. Adams RR, Wheatley SP, Gouldsworthy AM, et al: INCENP binds the Aurora-related kinase AIRK2 and is required to target it to chromosomes, the central spindle and cleavage furrow. Curr Biol 10: 1075-1078, 2000.

5. Ruchaud S, Carmena M and Earnshaw WC: Chromosomal passengers: conducting cell division. Nat Rev Mol Cell Biol 8: 798-812, 2007

6. Vagnarelli P and Earnshaw WC: Chromosomal passengers: the four-dimensional regulation of mitotic events. Chromosoma 113: 211-222, 2004

7. Platica O, Chen S, Ivan E, et al: PAR, a novel androgen regulated gene, ubiquitously expressed in normal and malignant cells. Int J Oncol 16: 1055-1061, 2000

8. Platica M, Verma RS, Macera MJ, et al: LNCaP-OM, a new androgen-resistant prostate cancer subline. In Vitro Cell Dev Biol Anim 33: 147-149, 1997

9. Hatakeyama S, Osawa M, Omine M, et al: JTB: a novel membrane protein gene at 1q21 rearranged in a jumping translocation. Oncogene 18: 2085-2090, 1999.

10. Platica M, Ivan E, Chen S, et al: Stable lower PAR expression decreased DU145 prostate cancer cell growth in SCID mice. Prostate 49: 200-207, 2001.

11. Platica M, Ivan E, Ionescu A, et al: Transformation of NIH 3T3 cells by enhanced PAR expression. Biochem Biophys Res Commun 314: 891-896, 2004

12. Platica M, Ivan E, Holland JF, et al: A pituitary gene encodes a protein that produces differentiation of breast and prostate cancer cells. Proc Natl Acad Sci USA 101: 1560-1565, 2004

13. Wheatley SP, Carvalho A, Vagnarelli P, et al: INCENP is required for proper targeting of Survivin to the centromeres and the anaphase spindle during mitosis. Curr Biol 11: 886-890, 2001

14. Altieri DC: Validating survivin as a cancer therapeutic target. Nat Rev Cancer 3: 46-54, 2003.

15. Altieri DC: The case for survivin as a regulator of microtubule dynamics and cell-death decisions. Curr Opin Cell Biol 18: 609-615, 2006

16. Skoufias DA, Mollinari C, Lacroix FB, et al: Human survivin is a kinetochore-associated passenger protein. J Cell Biol 151: 1575-1582, 2000.

17. Carmena M and Earnshaw WC: The cellular geography of aurora kinases. Nat Rev Mol Cell Biol 4: 842-854, 2003.

18. Giet R and Prigent C: Aurora/Ipl1p-related kinases, a new oncogenic family of mitotic serine-threonine kinases. J Cell Sci 112: 3591-3601, 1999

19. Meraldi P, Honda R and Nigg EA: Aurora kinases link chromosome segregation and cell division to cancer susceptibility. Curr Opin Genet Dev 14: 29-36, 2004.

20. Gassmann R, Carvalho A, Henzing AJ, et al: Borealin: a novel chromosomal passenger required for stability of the bipolar mitotic spindle. J Cell Biol 166: 179-191, 2004.

21. Mollinari C, Reynaud C, Martineau-Thuillier S, et al: The mammalian passenger protein TD-60 is an RCC1 family member with an essential role in prometaphase to metaphase progression. Dev Cell 5: 295-307, 2003

22. Bolton MA, Lan W, Powers SE, et al: Aurora B kinase exists in a complex with survivin and INCENP and its kinase activity is stimulated by survivin binding and phosphorylation. Mol Biol Cell 13: 3064-3077, 2002

23. Kaitna S, Mendoza M, Jantsch-Plunger V, et al: INCENP and an aurora-like kinase form a complex essential for chromosome segregation and efficient completion of cytokinesis. Curr Biol 10: $1172-1181,2000$.

24. Sampath SC, Ohi R, Leismann O, et al: The chromosomal passenger complex is required for chromatin-induced microtubule stabilization and spindle assembly. Cell 118: 187-202, 2004.

25. Vader G, Medema RH and Lens SM: The chromosomal passenger complex: guiding Aurora-B through mitosis. J Cell Biol 173: 833-837, 2006.

26. Vader G and Lens SM: The Aurora kinase family in cell division and cancer. Biochim Biophys Acta 1786: 60-72, 2008.

27. Castro A, Arlot-Bonnemains Y, Vigneron S, et al: APC/Fizzyrelated targets Aurora-A kinase for proteolysis. EMBO Rep 3: 457-462, 2002

28. Lindon C: Control of mitotic exit and cytokinesis by the APC/C Biochem Soc Trans 36: 405-410, 2008

29. Taguchi S, Honda K, Sugiura K, et al: Degradation of human Aurora-A protein kinase is mediated by hCdh1. FEBS Lett 519: $59-65,2002$
30. Fu J, Bian M, Jiang Q, et al: Roles of Aurora kinases in mitosis and tumorigenesis. Mol Cancer Res 5: 1-10, 2007.

31. Lens SM, Vader G and Medema RH: The case for Survivin as mitotic regulator. Curr Opin Cell Biol 18: 616-622, 2006.

32. Stenoien DL, Sen S, Mancini MA, et al: Dynamic association of a tumor amplified kinase, Aurora-A, with the centrosome and mitotic spindle. Cell Motil Cytoskeleton 55: 134-146, 2003.

33. Tsai MY, Wiese C, Cao K, et al: A Ran signalling pathway mediated by the mitotic kinase Aurora A in spindle assembly. Nat Cell Biol 5: 242-248, 2003

34. Katayama H, Brinkley WR and Sen S: The Aurora kinases: role in cell transformation and tumorigenesis. Cancer Metastasis Rev 22: 451-464, 2003.

35. Marumoto T, Hirota T, Morisaki T, et al: Roles of aurora-A kinase in mitotic entry and G2 checkpoint in mammalian cells. Genes Cells 7: 1173-1182, 2002.

36. Ferrand A, Chevrier V, Chauvin JP, et al: Ajuba: a new microtubule-associated protein that interacts with BUBR1 and Aurora B at kinetochores in metaphase. Biol Cell 101: 221-235, 2009.

37. Hirota T, Kunitoku N, Sasayama T, et al: Aurora-A and an interacting activator, the LIM protein Ajuba, are required for mitotic commitment in human cells. Cell 114: 585-598, 2003.

38. Eyers PA and Maller JL: Regulation of Xenopus Aurora A activation by TPX2. J Biol Chem 279: 9008-9015, 2004.

39. Han Z, Riefler GM, Saam JR, et al: The C. elegans Tousled-like kinase contributes to chromosome segregation as a substrate and regulator of the Aurora B kinase. Curr Biol 15: 894-904, 2005.

40. Kufer TA, Sillje HH, Korner R, et al: Human TPX2 is required for targeting Aurora-A kinase to the spindle. J Cell Biol 158: 617-623, 2002

41. Prigent $\mathrm{C}$ and Giet R: Aurora A and mitotic commitment. Cell 114: 531-532, 2003.

42. Crosio C, Fimia GM, Loury R, et al: Mitotic phosphorylation of histone H3: spatio-temporal regulation by mammalian Aurora kinases. Mol Cell Biol 22: 874-885, 2002.

43. Goto H, Yasui Y, Nigg EA, et al: Aurora-B phosphorylates Histone $\mathrm{H} 3$ at serine 28 with regard to the mitotic chromosome condensation. Genes Cells 7: 11-17, 2002.

44. Hsu JY, Sun ZW, Li X, et al: Mitotic phosphorylation of histone $\mathrm{H} 3$ is governed by Ipl1/aurora kinase and Glc7/PP1 phosphatase in budding yeast and nematodes. Cell 102: 279-291, 2000.

45. Hyman AA: Centrosome movement in the early divisions of Caenorhabditis elegans: a cortical site determining centrosome position. J Cell Biol 109: 1185-1193, 1989.

46. Lingle WL, Lukasiewicz K and Salisbury JL: Deregulation of the centrosome cycle and the origin of chromosomal instability in cancer. Adv Exp Med Biol 570: 393-421, 2005.

47. Meraldi P and Nigg EA: The centrosome cycle. FEBS Lett 521: 9-13, 2002.

48. Mullins JM and McIntosh JR: Isolation and initial characterization of the mammalian midbody. J Cell Biol 94: 654-661, 1982.

49. Steigemann P and Gerlich DW: Cytokinetic abscission: cellular dynamics at the midbody. Trends Cell Biol 19: 606-616, 2009.

50. Oliferenko $\mathrm{S}$ and Chew TG and Balasubramanian MK: Positioning cytokinesis. Genes Dev 23: 660-674, 2009.

51. Pollard TD: Progress towards understanding the mechanism of cytokinesis in fission yeast. Biochem Soc Trans 36: 425-430, 2008.

52. Adams RR, Carmena M and Earnshaw WC: Chromosomal passengers and the (aurora) ABCs of mitosis. Trends Cell Biol 11: 49-54, 2001.

53. Honda R, Korner R and Nigg EA: Exploring the functional interactions between Aurora B, INCENP, and survivin in mitosis. Mol Biol Cell 14: 3325-3341, 2003.

54. Jeyaprakash AA, Klein UR, Lindner D, et al: Structure of a Survivin-Borealin-INCENP core complex reveals how chromosomal passengers travel together. Cell 131: 271-285, 2007.

55. Ruchaud S, Carmena M and Earnshaw WC: The chromosomal passenger complex: one for all and all for one. Cell 131: 230-231, 2007.

56. Wong N, Chan A, Lee S-W, Lam E, To K-F, Lai PPB-S, Li XN Liew CT and Johnson PJ: Positional mapping for amplified DNA sequences on 1q21-q22 in hepatocellular carcinoma indicates candidate genes over-expression. J Hepatol 38: 298-306, 2003.

57. Kanome T, Itoh N, Ishikawa F, Mori K, Kim-Kaneyama J-R, Nose K and Shibanuma M: Characterization of jumping translocation breakpoint (JTB) gene product isolated as a TGF-B1inducible clone involved in regulation of mitochondrial function, cell growth and cell death. Oncogene 26: 5991-6001, 2007. 